\title{
The Past and Future of Clinical Biomechanics: Time to deliver on the legacy of pioneers such as Professor John P. Paul (1927-2013)
}

\author{
P.J. Rowe
}

\author{
Department of Biomedical Engineering, University of Strathclyde, Wolfson Centre, 106 \\ Rotten Row, Glasgow G4 ONW
}

\begin{abstract}
This editorial pays tribute to the work of Professor John P. Paul and his team at the University of Strathclyde in the 1960s and '70s, and subsequently by the Strathclyde Rehabilitation Engineering Group, as featured in the journal Medical Engineering \& Physics. It also includes a consideration of the nature of full biomechanical analysis of movement and how it can be mathematically modelled and physically recorded, the different approaches taken by Paul's and Winter's groups, respectively, and what a full biomechanical model should include in the future. The article also attempts to signpost the reader to future developments in the field, and how the techniques pioneered by Paul in the 1960s may influence Clinical Biomechanics and Rehabilitation in the years to come.
\end{abstract}

\section{Introduction}

Professor John P. Paul is rightly considered a pioneer of Biomechanics in the U.K. and across the world. I am proud to say he was my academic grandfather insofar as he was the supervisor of my Ph.D. supervisor Professor A. C. Nicol. He offered me my M.Sc. studentship in bioengineering, was head of department during my M.Sc. and Ph.D. in Bioengineering, was grant holder for my Ph.D. studentship and subsequent Research fellowship and chaired the first two sessions at international conferences at which I spoke. I distinctly remember at the second of these sessions talking to the Canadian speaker on before me who appeared very nervous and who said his nerves were down to Prof. Paul being the session chair. I said "but he's a nice guy to which he answered he maybe but he always ask the one question you don't want anyone to ask!" I realised then how lucky we in the biomechanics group at Strathclyde were to be subject, day in and day out, to John's extensive knowledge and questioning intelligence. It is a privilege for me to contribute this Editorial to accompany the 40th Anniversary Issue of Medical Engineering \& Physics, a journal to which Prof. Paul contributed 21 personal papers and his biomechanics group a further 61 . In this paper, I have attempted to summarise the legacy of pioneers of John's generation but also to apply the same critical questioning to the current state of clinical biomechanics as he might have done and offer some thoughts on the future of the subject for which he and subsequently I have had a lifelong passion.

\section{Background}

Professor Paul in his seminal thesis on "Forces at the Human Hip Joint" [1] paved the way for the development of successful hip joint arthroplasty. He was approached by the then Professor of Orthopaedic Surgery in Glasgow to research the topic because the implants they were using to try to repair the hip kept failing by breaking. It seemed logical to the clinicians that when in the stance phase of gait, i.e., standing on one leg and swinging the other, you would have slightly less than body weight (discounting the standing leg) pressing down on your hip; and allowing a bit extra for the thump of weight acceptance and the extra load at push off, then $1.5 \times$ body weight might be generated. $A$ 
lecturer in mechanical engineering at Strathclyde at the time (1963), John realised it was not as simple as this and he set about quantifying the true load on the hip joint scientifically. He borrowed and adapted a technique used in radiography called stereo-photogrammetry. This technique used two planar $\mathrm{x}$-rays from two orthogonal $\mathrm{x}$-ray cameras to recreate a three-dimensional model of the bony structure of some part of the body, for example, the pelvis. John realised that the mathematics and methods used in this technique could be adapted to reconstruct three-dimensional human movement.

He used two orthogonal cine cameras placed at right angles to each other to record the movement of the subject. He placed black and white markers on key anatomical locations while filming his subjects walking across the floor. He then had the films developed and projected them on a wall in order to digitise them. From the digitised locations of the anatomical markers in the two orthogonal planes he then used the trigonometrical methods developed in photogrammetry to recreate the location of the anatomical markers and hence the 6 degrees of freedom ( 3 translations and 3 rotations) of the foot, shank and thigh body segments of the lower limb and of the pelvis. He then joined these four segments together with suitable joints to form a four-segment, linked rigid-body model. He used a force plate to measure the Ground Reaction Force (GRF) between the modelled limb and floor and the GRF's location on the foot. He combined this data with the camera data to calculate the moments generated by the GRF about the three orthogonal axes at the hip. To these he added the moments generated by the weight of each segment below the hip by using anthropometric data to estimate both the location of that segment's centre of mass and its weight. Next the three inertia effects were added to the model for each segment below the hip. These were the moment generated around the hip due to the instantaneous acceleration of the thigh, shank and foot segments, the moment generated by the centripetal forces required to enable the centre of mass of each limb segment to rotate about its instantaneous centre of rotation and finally the moment necessary to cause each segment to rotate about its own centre of mass with its recorded angular acceleration.

Thus, the motion data, force plate data and anthropometric data could be combined in this way to calculate the total external moment generated at the hip for each frame of the movement. Using the principles of Newtonian mechanics for motion it can be seen that these total external moments calculated around each of the three orthogonal axes of the hip must be opposed by an equal and opposite moment around that hip axis generated by the internal body structures, primarily the muscles that cross the hip joint, or, according to D'Alembert, the sum of the moments at the joint (both external and internal) must add up to zero in all 3 planes.

Using this understanding Paul continued his analysis by constructing a 3D model of the musculature of the lower limb including points of origin and insertion of the muscles and calculation of their estimated moment arms around the relevant joints. He then solved this model in order to estimate the required muscle forces to stabilise each joint. Finally, he summated the internal muscle loads and the externally imposed forces to calculate the total load on the joint for each frame of the analysis. This is no easy analysis as there is considerable redundancy in the human body with 22 muscles crossing the hip and only 6 degrees of freedom at the hip. Paul realised that muscles can only provide tension (pull not push) and he assumed no antagonistic action in opposing muscle groups. He also lumped muscles together depending on their action (flexing, extending, abducting, adducting, internally rotating \& externally rotating) and provided an estimated moment arm for the group as a whole. In this way he was able to solve the problem for each frame of a gait cycle (on average 78 frames) for 18 gait cycles gathered from 9 male and 3 female subjects. He checked his solutions using an early EMG system.

This astonishing feat was achieved with only the most rudimentary assistance from a main frame computer and with all the equipment designed and validated by Paul himself. Paul's results indicated 
a range of peak hip joint loads of between 2.02 and 9.23 times body weight with a mean of 4,53 body weights. He estimated that at worst the amount of experimental error was $58 \%$ and the likely error was in practice $25 \%$ giving an average peak hip joint load estimated to be between 3.4 and 5.6 times body weight.

Paul also showed that the vast majority of the peak load experienced by the hip was due to the effect of the GRF about the hip combined with the body segment weights and the consequential actions of the muscles around the hip to stabilise the joint. In other words, there was a relatively small contribution to the peak load experienced by the hip joint contributed by the inertial effects of the body segments. To quote from Paul's thesis directly "it is also possible that a simplified analysis might be performed for the periods of maximum joint force considering only external force actions, and using the present measurement techniques". Paul's focus was on the load that would be placed on hip implants and how strong they needed to be to withstand that peak load and hence he suggested that to estimate the peak load the method could be simplified by ignoring the inertial effects.

An added bonus of this simplification is that the inertial effects are dependent on the first and second differentials of the displacements recorded by the camera system (velocity and acceleration both linear and angular). Calculating these differentials is notoriously difficult due to the induced noise of slope calculation (digital differentiation) and the data therefore requires filtering which can mask the rapid changes occurring. The filtering possible is also limited by the relatively low sampling frequencies $(25 \mathrm{~Hz}$ to $100 \mathrm{~Hz})$ available from the camera system-based displacements.

This simplified approach proposed by Paul was a pragmatic suggestion given the limitations of the technology available at the time but also relevant to the purpose to which the measurement process was targeted namely implant design in orthopaedics. This method became known as the "projection method" in which the force applied to the end of a limb is projected along its line of action and the moments it generates around a joint are calculated simply by the size of the GRF and the perpendicular distance between the line of action of the force and the joint centre. The weights of the segments acting at the centre of mass of each segment are included as are the moments they generate at the joints; however, the inertial effects are omitted. This analysis is often conducted in the three body planes and a biomechanical model of the joint including lines of action and points of application of joint contact forces, muscles and ligaments in each plane is used to calculate the set of internal forces required to stabilize the joint under these moments and at that joint angle using a set of simultaneous equations. This method is essentially quasi-static and assumes the segments are rigid, without inertia and the joints below the analysed joint are fused.

The projection method has been widely adopted in orthopaedic biomechanics since Paul's pioneering work. Paul's research group went on to computerise the technique and to use early TV cameras and infrared stroboscopes with reflective body markers to semi-automate the technique between 1970 and 1984. In 1984 the motion analysis company Vicon Ltd was founded in Oxford, UK as part of the Oxford metrics group of companies. Vicon produced a 4-camera version of the methodology drawing heavily on Paul's pioneering work and Vicon and Strathclyde have had a close relationship ever since. Vicon have gone on to develop and modernise the technology and methods and are now significant players in the life science, medical, engineering, animation and gaming sectors.

David Winter in his book "Biomechanics and Motor Control of Human Movement" (2005, 3rd edition) heavily criticised the projection method or as he called it the Floor Reaction Force Vector (FRFV) method. Winter was interested in the gait of children with cerebral palsy prior to surgery for improved functional mobility. For him the mechanics and motor control of the subject were both important, and he was focused not on the maximum loading on a joint but how the body mechanics were controlled throughout gait, how they were altered by cerebral palsy and how they might be improved by surgery.

An accepted author manuscript of the following editorial: Rowe, P. J. (2019). The past and future of clinical biomechanics: Time to deliver on the legacy of pioneers such as Professor J. P. Paul. Medical Engineering and Physics, 72, 66-69.

https://doi.org/10.1016/j.medengphy.2019.08.009 
In his book he includes a note about the wrong way to analyse moments of force (see page 105) in which he says "in the 1980's an erroneous technique has evolved and is still in use in the clinical area in spite of technical notes and letters to the editor. This technique is referred to as the FRFV approach ... and calculates the moments to be equal to the magnitude of the vector times the perpendicular distance between the joint centre and the vector".

Winter argued that while full biomechanical calculations including the mass-acceleration and moment of inertia-angular acceleration products were difficult and based on less than ideal data they were never the less vital when analysing gait and planning surgery. For example, they could often change the sign of the joint moment (e.g., from a flexing moment to an extending moment or from an adducting moment to an abducting moment) and hence change the consequences of transferring a muscle insertion during surgery. They also allowed for an analysis of the swing phase of gait when no moments are generated by the GRF and hence the movement and motor control of the swinging limb is entirely dependent on the inertial effects and weight of the segments and the stabilizing muscle moments. Winter therefore continued to advocate a full biomechanical model and used the camera data to estimate the inertial effects and include them in his analysis.

However, Winter's model [2] is itself incomplete as it does not include the centripetal force required to provide the instantaneous circular motion of the centre of mass of each segment around its instantaneous centre of rotation. Determination of the location of the instantaneous centre of rotation of each segment is itself difficult as it requires to use data from a number of frames to calculate the trajectory of the centre of mass, its radius of curvature in three-dimensional space and the centre of that radius of curvature in three dimensional space. Only once this has been done can the centripetal force required be included in the model for each segment. Although the centripetal force is relatively small it can be relevant in some biomechanical situations such as the swing phase of the above knee amputee where it provides a distraction force on the residual limb segment. If left unopposed this would pull the artificial leg from the stump and hence suspension of the artificial limb by vacuum formation in the socket or by suspension straps is required to prevent this from occurring. A fully comprehensive, responsive but accurate biomechanical model of human walking which could be applied in all these domains has yet to be developed.

As has been discussed motion capture systems based on cameras can accurately locate and orientate body segments but struggle to calculate velocities and accelerations accurately when operating at the typical sampling frequencies of 25,50 or $100 \mathrm{~Hz}$ and hence they on their own poorly represent the inertial effects of movement. High speed motion capture cameras operating at $1000 \mathrm{~Hz}$ are available and could do the job but are prohibitively expenses for multi-camera systems. Inertial Measurement Units (IMUs) on the other had can sample acceleration (with the accelerometers) and angular velocity (with the gyroscopes) at high speed so providing estimates of the inertial effects. However, these devices suffer from drift and integration errors making their ability to track body segment's global position and orientation on their own difficult over a period of time. If the segment is stationary for a short period of time the magnetometers in the IMU can be used to reset orientation in space. However, in some applications, for example walking on a treadmill, long periods can elapse when the IMU is not stationary and hence no correction can be applied. For global position the situation is worse and the global position cannot be reset by IMU sensor fusion. The IMU is only able to predict its current position based on its starting position and the double integration of the acceleration, information which is prone to drift over time and a double integral also prone to increasing error with time. The result is that IMU only systems can accurately record velocities and accelerations. Global position and orientation remain a challenge to monitor over time.

A solution to these issues would be to use both motion camera data and IMU data captured simultaneously. This could be achieved using a segment marker cluster (such as the Vicon Pulsar active 
LED marker cluster) and an IMU (such as the Vicon Bluethunder IMU) co-located on the segment. It is possible for the IMU to operate at much higher frequencies than the motion capture, say, at $1000 \mathrm{~Hz}$, hence reducing the accuracy issues of these devices. We have explored the requirements for fully characterising the biomechanics of a body segment in this way and have found it to be feasible and to lead to an improvement in the accuracy of the estimation of the inertial forces and moments of a moving segment. We have conducted experiments using a pendulum, a robotic arm and in humans and have shown proof of concept for this approach.

An even better solution would be to build the IMU into the cluster and produce a cluster/IMU combo that is capable of providing real time data fusion. Vicon are currently developing such a cluster and the software and data transfer processes to go with it. This new technology will allow cluster/IMU data fusion and allow us to provide for the first time an accurate, responsive and reliable fully complete biomechanical model of human motion which includes both externally imposed and inertially generated forces and moments in the solution. Such as system would fulfil the needs of both Paul and Winter and many others in the biomechanics research community.

It is surprising to me, and I think it would be disappointing to both Paul and Winter, that despite the pioneering work of their generation, clinical biomechanics has found surprisingly little application in clinical practice. The one exception seems to be in the treament of cerebral palsy, where threedimensional, whole-body, full-gait analysis has become a standard of clinical care prior to surgery. However, this is the exception rather than the rule. Even in the assessment of novel health technologies there has been a reluctance to adopt clinical biomechanical methods. For example, in knee joint arthroplasty, where we are replacing the bearing in the joint, it is still very rare for assessors to actually measure with motion capture how that new bearing articulates during functional activity. Knee joint arthroplasty tends to be evaluated using questionnaires focused on pain, deformity, complications and general functional ability such as the ability to walk rather than how the new bearing articulates during functional activities. Indeed, a knee joint fusion would score very well in many of these assessments.

Another similar area is the provision of feedback during rehabilitation where mirrors or video cameras maybe used but motion capture is not. Mirrors show everything reflected and one only has to try to knot a tie in a mirror to appreciate how inappropriate this form of feedback can be for motor learning. Video is better and becoming ubiquitous with the use of mobile phones but is from one point of view only, cannot provide accurate joint kinematics and is difficult to anonymise, a growing concern in these days of digital security and privacy concerns. The failure of the pioneering work of Paul and his generation to penetrate the mass market of clinical biomechanics and rehabilitation is in my opinion due to two main factors the price of the systems and their usability in clinical practice.

If made sufficiently inexpensive and easy to use, it is my opinion that full biomechanical motion analysis systems using combined camera and IMU data could find widespread use in clinical biomechanics not only in measuring the outcome of health interventions for research but also in diagnostics, prescription and tuning of prosthetics and orthotics and in the important and growing area of visual feedback for motor relearning. Sports biomechanics has shown the importance of knowledge of performance and knowledge of results in motor learning and has used biomechanical data presented visually to the user to improve task performance for over a decade now leading to record breaking performances. The same principles could be applied in clinical rehabilitation.

Motion capture will soon become commonplace on the high street with virtual reality-based group gaming activities already taking off. This technology by necessity needs to be simple to use and rapid to apply to meet the needs of this commercial sector. It will also be less expensive given this mass market. It is likely therefore that the same technology will be available for clinical rehabilitation. One 
can envisage a situation where a user undertaking rehabilitation could enter a bespoke gym, could apply the cluster/IMU combos to their relevant body segments, could be picked up and labelled automatically by a multi-camera motion analysis system attached to the walls of the gym and they could then receive visual feedback on a screen or heads up display of their biomechanical performance as they undertake their rehabilitation regime. Indeed, each client could receive bespoke knowledge of performance and results for their particular tasks, at their stage of rehabilitation and in their preferred format. In this way motion capture could truly become a mainstream activity effecting in a positive way the lives of many, something which I know, were he alive today, would greatly appeal to Professor Paul. This is a significant focus of my research team and I would urge others to consider it also. Professor Paul was generous in his sharing of ideas and inspiring others. I have tried in this article to mirror this spirit and would welcome collaboration from any readers of this article who share the vision presented for the future of clinical biomechanics and passion for the subject that he helped inspire in me.

\section{References}

[1] John P. Paul

Forces at the human hip joint

$\mathrm{PhD}$ thesis

(1967)

http://theses.gla.ac.uk/3913/

[2] David A. Winter

Biomechanics and Motor Control of Human Movement

(Third Edition), John Wiley and sons, New Jersey, USA (2005)

ISBN 0-471-44989-X 
Editor Note: Below is the list of outputs authored by Professor J.P. Paul and co-workers, and published in Medical Engineering \& Physics (1981-1993) and Journal of Biomedical Engineering (1994 - 2010), in reverse chronological order. The list is to accompany the editorial written by Philip Rowe, to appear on ScienceDirect as a supplement to the Virtual Special Issue with the same title as above.

1. Abu Osman, N. A., Spence, W. D., Solomonidis, S. E., Paul, J. P. and Weir, A. M. (2010) 'The patellar tendon bar! Is it a necessary feature?', Medical Engineering \& Physics, 32(7), pp. 760-765.

2. Paul, J. P. (2008) 'Loss in mechanical contact of cement-bearing: A biomechanical model', Medical Engineering \& Physics, 30(5), pp. 669-669.

3. Paul, J. P. (2007) 'Measurement of local strains induced into the femur by trochanteric Gamma nail implants with one or two distal screws', Medical Engineering \& Physics, 29(10), pp. 1179-1179.

4. Paul, J. P. (2004) 'Intremedullary femoral nails: one or two lag screws? A preliminary study', Medical Engineering \& Physics, 26(4), pp. 359-359.

5. Paul, J. P. (2003) 'Optimizing the biomechanical compatibility of orthopedic screws for bone fracture fixation', Medical Engineering \& Physics, 25(5), pp. 435-435.

6. Paul, J. P. (2001b) 'Re: Influence of head constraint and muscle forces on the strain distribution within the intact femur', Medical Engineering \& Physics, 23(6), pp. 435-435.

7. Paul, J. P. (2001a) 'Comparison of computational analysis with clinical measurement of stresses on a below-knee residual limb in a prosthetic socket Medical Engineering \& Physics 22 (2000):607-12', Medical Engineering \& Physics, 23(7), pp. 519-519.

8. Paul, J. P. (1998b) 'Letter to the editor', Medical Engineering \& Physics, 20(3), pp. 229-229.

9. Paul, J. P. (1998a) 'Codman's Paradox of the arms rotation is not a paradox: mathematical validation by Julio C Politti, Gustavo Goroso, Max E. Valentinuzzi, Orlando Bravo. Medical Engineering and Physics, 20(4): 257-60,1998', Medical Engineering \& Physics, 20(8), pp. 639639.

10. Draper, E. R. C., Strachan, R. K., Hughes, S. P. F., Nicol, A. C. and Paul, J. P. (1997) 'The design and performance of an experimental external fixator with variable axial stiffness and a compressive force transducer', Medical Engineering \& Physics, 19(8), pp. 690-695.

11. Paul, J. P. (1997) 'Stress and strain distribution within the intact femur: Compression or bending?', Medical Engineering \& Physics, 19(1), pp. 97-97.

12. Paul, J. P. (1996) 'Three-dimensional finite element stress analysis of the polypropylene ankle-foot orthoses: Static analysis', Medical Engineering \& Physics, 18(7), pp. 607-607.

13. Draper, E. R. C., Wallace, A. L., Strachan, R. K., Hughes, S. P. F., Nicol, A. C. and Paul, J. P. (1995) 'THE DESIGN AND PERFORMANCE OF AN EXPERIMENTAL EXTERNAL FIXATION DEVICE WITH LOAD TRANSDUCERS', Medical Engineering \& Physics, 17(8), pp. 618-624.

14. Paul, J. P. (1993) 'UNIVERSAL JOINT SLIPPAGE AS A CAUSE OF HOFMANN HALF-FRAME EXTERNAL FIXATOR FAILURE', Journal of Biomedical Engineering, 15(1), pp. 83-83.

15. Paul, J. P. (1992) 'BENDING AND FRACTURE OF THE FEMORAL COMPONENT IN CEMENTED TOTAL HIP-REPLACEMENT - COMMENT', Journal of Biomedical Engineering, 14(5), pp. 444444.

16. Badran, A. K., Fisher, A. C., Durrani, T. S. and Paul, J. P. (1991) 'AN AUTOMATIC MATCHING TECHNIQUE FOR PATIENT ALIGNMENT', Journal of Biomedical Engineering, 13(4), pp. 281286.

17. Badran, A. K., Durrani, T. S., Fisher, A. C. and Paul, J. P. (1990) 'PATIENT REALIGNMENT IN MAGNETIC-RESONANCE-IMAGING OF THE HEAD - AN ALGORITHM USING MATHEMATICAL MORPHOLOGY FOR FEATURE-EXTRACTION', Journal of Biomedical Engineering, 12(2), pp. 138-142. 
18. Andrews, B. J., Baxendale, R. H., Barnett, R., Phillips, G. F., Yamazaki, T., Paul, J. P. and Freeman, P. A. (1988) 'HYBRID FES ORTHOSIS INCORPORATING CLOSED-LOOP CONTROL AND SENSORY FEEDBACK', Journal of Biomedical Engineering, 10(2), pp. 189-195.

19. Pournezam, M., Andrews, B. J., Baxendale, R. H., Phillips, G. F. and Paul, J. P. (1988) 'REDUCTION OF MUSCLE FATIGUE IN MAN BY CYCLICAL STIMULATION', Journal of Biomedical Engineering,10(2), pp. 196-200.

20. Paul, J. P. (1986) 'MINIATURE LOAD CELLS FOR THE MEASUREMENT OF FOOT GROUND REACTION FORCES AND CENTER OF FOOT PRESSURE DURING GAIT', Journal of Biomedical Engineering, 8(4), pp. 367-367.

21. Brown, T. R. M., Paul, J. P., Kelly, I. G. and Hamblen, D. L. (1981) 'BIOMECHANICAL ASSESSMENT OF PATIENTS TREATED BY JOINT SURGERY', Journal of Biomedical Engineering, 3(4), pp. 297-304. 